\title{
T-rays in biomedicine and security
}

\author{
T. Rainsford, Member, IEEE, G.M. Png, Student Member, IEEE, W. Withayachumnankul, \\ B. Ferguson, Member, IEEE, S.P. Mickan, Member, IEEE and D. Abbott, Fellow, IEEE
}

(Invited Paper)

\begin{abstract}
With a number of commercial systems becoming increasingly available, and improved generation and detection techniques T-ray systems are increasingly finding new applications. One of the main technical challenges is to make systems low cost, compact and easy to use. In this paper we suggest some novel solutions for parameter extraction, image enhancement, improved signal-to-noise ratio and classification schemes. These tools are useful in a number of biomedical and security applications.
\end{abstract}

Index Terms-T-rays, terahertz, time-domain spectroscopy, Fabry-Pérot removal

Various rotational, vibrational and translational modes of molecules are within the T-ray range $(0.1-10 \mathrm{THz})$. Since these modes are unique to a particular molecule it is possible to obtain a "THz ngerprint" allowing for the identi cation of chemical substances. Since T-rays can see through paper envelopes they can be employed by mail sorters to check for biological and chemical hazards for example, in the detection of anthrax [1] [2]. Research has already shown that THz pulsed spectroscopy can probe the physical state of a pharmaceutically active ingredient [3], paving the way for quality-control monitoring at various intermediate steps in the manufacturing process rather than just at the end point. $\mathrm{THz}$ spectroscopy allows not only for exploration of molecular structures but of molecular dynamics [4].

We have carried out preliminary testing of the sensitivity of T-ray Differential Time Domain Spectroscopy (DTDS) to thin layers of bound biomolecules [5]. T-ray signals were observed for biotin molecules bound to an avidin sensor, avidin molecules bound to a biotin sensor, bead-enhanced avidin bound to biotin, and the explosive trinitrotoluene (TNT) bound to TNT antibodies. It was found that agarose beads attached to the avidin amplify the target layer thickness, potentially increasing the biosensor's signal. Figure 1 shows that the Tray DTDS waveforms are suf cient to distinguish between the bound and unbound states of the biosensor indicating the potential utility of a T-ray-based biosensor.

Apart from spectroscopic characterisation, T-rays can also provide X-ray-like density images, but without depositing harmful amounts of energy in human soft tissue. The most extensive study of the application of THz-TDS to cancer detection has been conducted by researchers are the University of Cambridge and TeraView Limited [6], [7]. These studies have focused on excised tissue where a number of

T. Rainsford, G.M. Png, W. Withayachumnankul, B. Ferguson, S.P. Mickan and D. Abbott are with the Department of Electrical \& Electronic Engineering, The University of Adelaide, SA 5005, Australia (e-mail: tamath@eleceng.adelaide.edu.au). B. Ferguson is now with Tenix Defence Pty Ltd, Mawson Lakes, SA 5090, Australia and W. Withayachumnankul is also with Department of Information Engineering, Faculty of Engineering, King Mongkut's Institute of Technology Ladkrabang, Bangkok 10520, Thailand

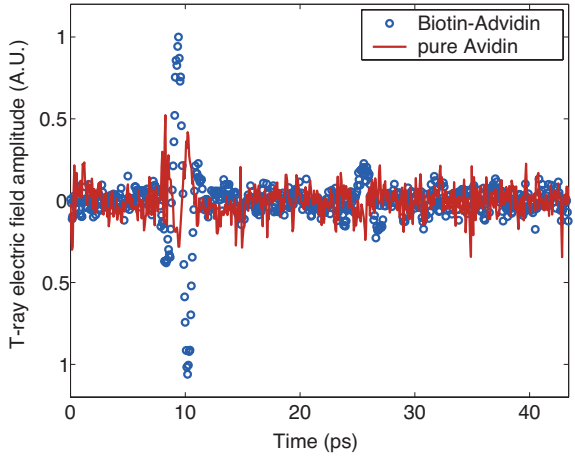

Fig. 1. Two time-domain scans are made, one to test for the presence of an avidin (concentration of avidin is less than $15 \mathrm{ng} / \mathrm{cm}^{2}$ ) layer ('biotin-avidin') and one to con rm that the measurements are due solely to the avidin layer.

factors in uence the measured $\mathrm{THz}$ response; changes in the concentration of water molecules and its bonds are likely to dominate the response. We have investigated the problem at a cellular level, isolating the cells' responses from that of bulk tissue properties. We use a classi cation framework that allows automatic differentiation between different cell types. Statistical analysis is then applied to analyse the $\mathrm{THz}$ spectral results. These techniques are able to differentiate between the $\mathrm{THz}$ responses of cancerous and normal human bone cells with high accuracy. The Mahalanobis distance was used to classify the responses. The input features to the classi er were the deconvolved amplitude and phase at speci c frequencies. It was not possible to manually choose optimal frequencies, so they were chosen using a genetic algorithm that identi ed nearoptimal frequencies. The genetic algorithm resulted in a set of near-optimum training features which provided a classi cation accuracy of $98.6 \%$, as can be seen in Figure 2. The results from this preliminary case study are promising: they show that THz-TDS can detect the response of a thin layer of cells with a thickness of under $100 \mu \mathrm{m}$. They also show that there is suf cient spectral signature information to allow a classi er to be trained to recognise speci c types of cells.

Although plots of time versus terahertz amplitude, and frequency versus terahertz magnitude are some of the most common ways of analyzing terahertz data, no standard rendering technique has been established. One solution [8] to $\mathrm{THz}$ imaging is to implement a new form of phase contrast imaging inspired by Zernicke's optical phase contrast method: when light passes through free-space/air (surround path), the phase and amplitude information in the light wave is unaltered. When light passes through a phase object (particle path), the amplitude of the light wave is slightly attenuated due to energy 


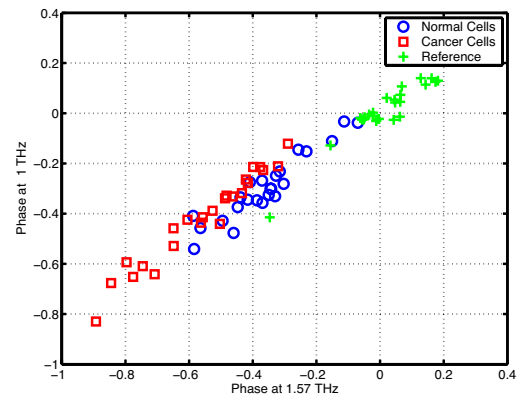

Fig. 2. Scatterplot of the deconvolved phase of human osteosarcoma cells and normal human bone cells shown at frequencies of 1.00 and $1.57 \mathrm{THz}$.

loss. The time delay experienced by the ray is affected by the thickness and refractive index of the medium, while the amplitude attenuation tells us how absorptive (opaque) the medium is. The goal of optical phase contrast is to align the surround and particle waveforms so that they are in phase and can recombine constructively in time. The specimen's opacity to T-rays can be obtained by calculating the amplitude difference of the pro les with respect to the reference. Rendering of these extracted data provides a novel form of phase contrast imaging. This combined opacity and optical path length information enables rendering of the data as a 3Dimensional image in applications such as MATLAB, enabling the viewer two additional dimensions when examining the terahertz data (Figure 3).

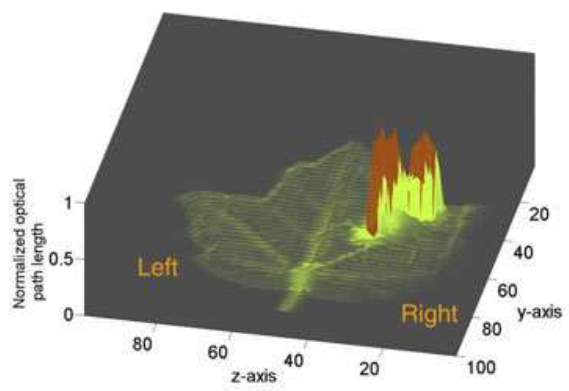

Fig. 3. Phase contrast image of an insect on a leaf, containing both optical path length and opacity information. Smaller veins are visible because of their low opacity values. The insect has high opacity implying it contains a fair amount of moisture that absorbed the T-rays.

So far there is no direct and ef cient method to discern the re ections caused by the Fabry-Pérot effect from a detected T-ray signal. One of the existing methods is to window the signal in the spatial domain [9]. However, it requires prior knowledge of sample thickness, refractive indices, and pulse width to precisely locate the position of re ections. Besides, it cannot distinguish the re ections when the primary pulse possesses long uctuations. Other methods deal with the re ections along with the parameter extraction process [9], [10], [11], where ef cienc y is degraded by iterative techniques. We have proposed a method that locates and discriminates the re ections from the required signal directly [12]. By tting polynomials to the logarithm and the argument of the transfer function, the re ections can be estimated and subtracted from

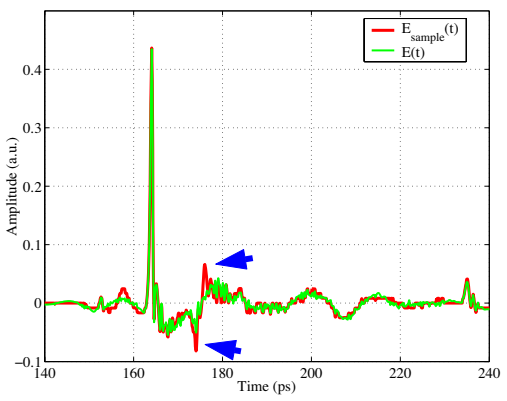

Fig. 4. Probing terahertz signals for a silicon wafer before and after FabryPérot removal is carried out. The arrows indicate reduced amplitude of the pulse.

the probing signal, leaving only the primary pulse. Since the method is performed in the frequency domain, it can correctly remove the re ections regardless of the sample thickness or material parameters. Moreover, no iterative procedure is required by the process, thus leading to ef cient real-time application. The method performs successfully on the test sample, even if the noise or the uctuation of the primary pulse perturbs the temporal location and amplitude of the re ections (see for example, Figure 4).

\section{REFERENCES}

[1] M. K. Choi, A. Bettermann, and D. W. van der Wiede, "Potential for detection of explosive and biological hazards with electronic terahertz systems," Phil. Trans. R. Soc. Lond. A, vol. 362, pp. 337-349, 2004.

[2] B. Ferguson, S. Wang, D. Abbott, and X. C. Zhang, "Powder retection with THz imaging," Proc. SPIE Terahertz for Military and Security Applications, vol. 5070, pp. 7-16, 2003.

[3] P. F. Taday, I. V. Bradley, D. D. Arnone, and M. Pepper, "Using Terahertz pulse spectroscopy to study the crystalline structure of a drug: a case study of the polymorphs of ranitidine hydrochloride," J. Pharm. Sci., vol. 92, pp. 831-838, 2003.

[4] F. De Lucia, "Spectroscopy in the terahertz spectral region," in Sensing With Terahertz Radiation, D. Mittleman, Ed. Springer Verlag, Berlin, 2003, pp. 39-115.

[5] S. P. Mickan, A. Menikh, H. Liu, C. A. Mannella, R. MacColl, D. Abbott, J. Munch, and X. C. Zhang, "Label-free bioaf nity detection using terahertz technology," Physics in Medicine and Biology, vol. 47, no. 21 , pp. 3789-3795, 2002

[6] R. M. Woodward, B. E. Cole, and V. P. Wallace, "THz pulse imaging of in vitro basal cell carcinoma samples," TOPS, vol. 56, pp. 329-330, 2001.

[7] — , "THz pulse imaging in re ection geometry of human skin cancer and skin tissue," Physics in Medicine and Biology, vol. 47, no. 21, pp. 3853-3863, 2002.

[8] G. M. Png, S. P. Mickan, T. Rainsford, and D. Abbott, "Investigation of phase contrast methods for terahertz imaging," Proc. SPIE Smart Structures, Devices and Systems II, vol. 56449, 2005.

[9] L. Duvillaret, F. Garet, and J.-L. Coutaz, "A reliable method for extraction of material parameters in terahertz time-domain spectroscopy," IEEE Journal of Selected Topics in Quantum Electronics, vol. 2, no. 3, pp. 739-746, 1996.

[10] L. Duvillaret, F. Garet, and J. L. Coutaz, "Highly precise determination of optical constants and sample thickness in terahertz time-domain spectroscopy," Applied Optics, vol. 38, no. 2, pp. 409-415, 1999.

[11] T. D. Dorney, R. G. Baraniuk, and D. M. Mittleman, "Material parameter estimation with terahertz time-domain spectroscopy," Journal of the Optical Society of America A, vol. 18, no. 7, pp. 1562-1571, 2001.

[12] W. Withayachumnankul, B. Ferguson, T. Rainsford, S. P. Mickan, and D. Abbott, "Direct Fabry-Pérot effect removal," submitted to Fluctuation and Noise Letters. 